\title{
Modelamiento de la precipitación química mediante la ecuación de balance poblacional
}

\section{Modeling chemical precipitation using the population balance equation}

\author{
Salvador T. Oré \\ (1) Facultad de Ingeniería Química, Universidad Nacional del Centro del Perú \\ Email: salvadorore@gmail.com
}

\section{Resumen}

El presente trabajo de investigación tiene como objetivos desarrollar la ecuación del balance poblacional para el proceso de precipitación, y resolver el modelo matemático aplicando el método reticular de Boltzmann contrastándolos con otros métodos. En teoría de probabilidad, la distribución de una variable aleatoria es una función que asigna a cada suceso definido sobre la variable aleatoria la probabilidad de que dicho suceso ocurra. Este concepto tiene una aplicación en los BP, se puede usar la distribución de probabilidad conocida como función de distribución para cuantificar las partículas en cada uno de los tamaños que pueda alcanzar el cristal, es decir con una función de distribución se puede ponderar la cantidad de cristales que tienen cada uno de los tamaños en los que se ha discretizado el tamaño de cristal ( $L$ ). El argumento básico para el uso de estos tipos de métodos cinéticos simplificados en la simulación de flujos de fluidos macroscópicos es el de que la dinámica macroscópica de un fluido es el resultado de la conducta colectiva de muchas partículas microscópicas en el sistema y que la dinámica macroscópica no es sensible a los detalles subyacentes en la física microscópica. Mediante el desarrollo de una versión simplificada de las ecuaciones cinéticas complejas, como lo es la ecuación de Boltzmann, es posible evitar que sigue a cada partícula como en simulaciones de dinámica molecular. Se concluye que la ecuación del balance poblacional en una dimensión ( I D) es desarrollada para un proceso de precipitación o cristalización reactiva, considerando el crecimiento, la nucleación, la agregación y la rotura; y el Método Reticular de Boltzmann resuelve el sistema de ecuaciones del balance poblacional el cual ha sido contrastado con los modelos analíticos para la precipitación del nitrato de potasio.

\begin{abstract}
This research aims to develop the population balance equation for the precipitation process and solve the mathematical model using the lattice Boltzmann method contrasting them with other methods. In probability theory, the distribution of a random variable is a function that assigns to each defined event on the random variable the probability that the event will occur. This concept has application in BP, can use the probability distribution known as distribution function to quantify the particles in each of the sizes that can reach the glass, a distribution function can weigh the amount of crystals each having sizes in which discretized crystal size ( $L$ ). The basic argument for the use of these types of kinetic methods simplified in flow simulation macroscopic fluid is that the macroscopic dynamics of a fluid is the result of the collective behavior of many microscopic particles in the system and macroscopic dynamics it is not sensitive to the underlying details microscopic physics. By developing a simplified version of the complex kinetic equations, as is the Boltzmann equation, it is possible to avoid that follows each particle and molecular dynamics simulations. It is concluded that the population balance equation in one dimension ( I D) is developed for a precipitation process or reactive crystallization, considering the growth, nucleation, aggregation and breakage; and Lattice Boltzmann Method solves the system of equations of population balance which has been contrasted with analytical models for precipitation of potassium nitrate.
\end{abstract}

Keywords: population balance equation

Palabras clave: ecuación balance poblacional 


\section{Introducción}

Los procesos con partículas se encuentran entre los más comunes en el procesamiento químico. Se requieren técnicas para la generación precisa y eficiente de soluciones a las ecuaciones que rigen para el diseño y el control de estos sistemas. Las diferentes exigencias de calidad de la solución y la velocidad entre las aplicaciones y los sistemas de diversidad específica requieren una cuidadosa consideración en la elección de técnicas numéricas apropiadas.

Los procesos de precipitación química, están ligados a los procesos de remediación de los drenajes de los procesos industriales, y que, a través del tiempo, se han limitado a la separación de los agentes contaminantes, siendo estos almacenados, y convirtiéndose en potenciales agentes contaminantes, dado a los procesos de reversibilidad.

Una nueva tendencia es la reutilización de estos agentes ya sea por ejemplo los metales pesados, a partir de los cuales es factible la utilización de otros productos como por ejemplo los pigmentos. Pero este proceso requiere una investigación sobre el comportamiento, y particularmente en este caso es sobre su modelamiento. El proyecto presentado sobre el "Modelamiento de la precipitación química mediante la ecuación de balance poblacional", tiene como propósito el de desarrollar un modelamiento en base al balance poblacional para estudiar el comportamiento del proceso de precipitación química, cuyas ecuaciones en derivadas parciales serán resueltas aplicando el método reticular de Boltzmann, cuyos resultados serán contrastados con datos experimentales.

\section{Objetivo general:}

Realizar el modelamiento del proceso de precipitación utilizando la ecuación del balance poblacional.

\section{Objetivos específicos:}

- Desarrollar la ecuación del balance poblacional para el proceso de precipitación.

- Resolver el modelo matemático aplicando el método reticular de Boltzmann contrastándolos con datos experimentales.

\section{Materiales y métodos}

Dentro de los métodos numéricos para resolver las ecuaciones de balance poblacional sobresalen los métodos de momentos tales como método de cuadratura de momentos, (QMOM), método de cuadratura de momentos directo (DQMOM), método de cuadratura de momento seccional (SQMOM). En la presente investigación, desarrollaremos el método reticular de Bolltzmann.

\section{Método Reticular de Boltzmann}

Métodos basados en redes han recibido una atención significativa en las últimas dos décadas para la simulación hidrodinámica. La principal diferencia entre el método basado en redes y otros métodos tradicionales, por ejemplo, el método de diferencias finitas, es que la mayoría de los métodos tradicionales siguen un enfoque de arriba hacia abajo, donde las ecuaciones que rigen las diferenciales parciales (PDE) en la escala macroscópica se discretizan para obtener ecuaciones diferencial ordinaria (EDOs) o ecuaciones algebraicas. Por otro lado, los métodos basados en redes adoptan un enfoque de abajo hacia arriba, donde en lugar de utilizar discretización cinética de pseudo-partículas se utiliza de una manera tal que las ecuaciones que gobiernan se recuperan a la escala apropiada. LBM fue desarrollado inicialmente como un método conocido como Gas Autómatas de Gas Entramado (LGCA). La característica distintiva clave de la LBM es la introducción de la ecuación de Boltzmann para superar el problema del ruido estadístico encontrado en LGCA. Con sus raíces en la teoría cinética, LBM utiliza una representación simplificada del estado microscópico del proceso de modo que el modelo es computacionalmente viable y puede capturar la descripción esencial del sistema a nivel macroscópico. LBM ahora se ha establecido como un esquema popular debido a su capacidad de proporcionar el código rápido, fácil implementación y versatilidad. Se ha aplicado a diversos problemas, incluyendo la hidrodinámica, la turbulencia, el flujo de múltiples fases, microflujo, los fluidos no newtonianos y cristalización. Varias críticas de LBM están disponibles en la literatura.

PBE con la agregación y la rotura es análoga a la ecuación de advección con término fuente. Así, en el debate posterior, primero derivamos LBM para la ecuación de advección con término fuente y luego aplicamos el esquema desarrollado para resolver PBES con la agregación y la rotura mediante la identificación de sus similitudes más tarde en el papel.

\section{Principio}

La ecuación de advección con un término fuente se puede escribir como:

$$
\frac{\partial \rho}{\partial t}+\frac{\partial(v \rho)}{\partial r}=F
$$

donde:

$\rho=$ Concentración de la especie transportada (escalar pasivo)

$\vee=$ Velocidad de advección y

$F=$ Término fuente.

Con el fin de resolver esta ecuación de advección, algunas partículas ficticias, que se asemejan grupos de moléculas, se consideran en la escala mesoscópica. Mientras que las moléculas se mueven al azar en el espacio, estas partículas ficticias están restringidos a moverse con ciertas velocidades, la cual se eligen cuidadosamente de manera que están consistente tienda de campaña con la simetría y el requisito de isotropía de la dinámica ma- 
croscópicas. Encontrar un conjunto de velocidades de este tipo implica un procedimiento de ensayo y error. Uno comienza típicamente con un conjunto de velocidades en un enrejado basado en consideraciones de cálculo, y comprueba si es posible construir un modelo, cuya solución a gran escala a largo plazo coincide con la ecuación macroscópica. En la forma discreta, el modelo más simple posible tiene tres tipos de partículas ficticios con velocidades:

$$
c_{i}=\{0, c,-c\}
$$

es decir, estacionaria (0), a la derecha en movimiento $(+)$, a la izquierda en movimiento (-).

Con el fin de resolver la ecuación. (3.I) con LBM, la siguiente ecuación cinética con la fuerza (fuente) se utiliza plazo:

$$
\begin{array}{r}
\frac{\partial f_{i}}{\partial t}+c_{i} \frac{\partial f_{i}}{\partial r}=-\frac{1}{\tau}\left(f_{i}\right. \\
\left.-f_{i}^{e q}\right) \\
+F_{i} \\
i=0,1,2
\end{array}
$$

$\tau>0=$ Tiempo de relajación,

fi = Función de distribución discreta de Boltzmann,

$\mathrm{fi}^{\mathrm{eq}}=$ Función de distribución discreta Boltzmann equilibrio,

ci $=$ Velocidad discreto y

fi $=$ Término de fuerza discreta.

En la forma más simple puede ser tomado como

fi $=$ wiF(Dawson et al., 1993), donde

wi $=$ Peso asociado con velocidad ci

En la Ec. (3.5), los subíndices 0 , I y 2 se refieren a estacionaria, justo el movimiento y dejan partículas en movimiento, respectivamente. El lado izquierdo de la ecuación. (3.3) denota el vuelo libre, mientras que el lado derecho representa la relajación de las partículas de equilibrio aproximación por colisión. Las distribuciones de equilibrio se pueden encontrar minimizando apropiada función de entropía sujeto a las limitaciones de masa y de la conservación del momento. La forma discreta de la función de entropía correspondiente, también conocida como función de $\mathrm{H}$

$$
H=\sum_{i=0}^{2} f_{i}(\ln -1)
$$

Para la ecuación de advección ID, los pesos pueden ser seleccionados como

$$
\begin{aligned}
& w_{0}=4 / 6 \\
& w_{1}=1 / 6 \\
& w_{2}=1 / 6
\end{aligned}
$$

Las limitaciones para la conservación local de la masa y cantidad de movimiento se dan como:

$$
\begin{aligned}
& \sum_{i=0}^{2} f_{i} \\
& =f_{0}+f_{1}+f_{2} \\
& =\rho \\
& \sum_{i=0}^{2} c_{i} f_{i} \\
& =c\left(f_{1}-f_{2}\right) \\
& =\rho u
\end{aligned}
$$

Donde

$u=$ velocidad media local

Al resolver el problema de minimización, obtenemos los valores de equilibrio de la función de distribución discreta Boltzmann como:

$$
\begin{aligned}
& f_{0}^{e q}(\rho, u) \\
& =\frac{2 \rho}{3}[2 \\
& \left.-\sqrt{1+u^{2} / c_{s}^{2}}\right] \\
& f_{1}^{e q}(\rho, u) \\
& =\frac{\rho}{3}\left[\left(u c-c_{s}^{2}\right) / 2 c_{s}^{2}\right. \\
& \left.+\sqrt{1+u^{2} / c_{s}^{2}}\right] \\
& f_{2}^{e q}(\rho, u) \\
& =\frac{\rho}{3}\left[\left(-u c+c_{s}^{2}\right) / 2 c_{s}^{2}\right. \\
& \left.+\sqrt{1+u^{2} / c_{s}^{2}}\right],
\end{aligned}
$$

Donde $\mathrm{C}_{\mathrm{s}}=\mathrm{C}_{3} 3$ es análoga a la velocidad del sonido en el sistema. La conservación de la masa y en el impulso durante la colisión se recuperará la ecuación de Navier-Stokes en el límite de escala largo de mucho tiempo, mientras que la conservación de la masa sólo se 
recuperará la ecuación de advección. Dado que estamos interesados en la ecuación de advección, distribución de equilibrio y se calcula en la velocidad dada $u=v$. Se muestra a continuación que romper esta conservación impulso conduce a la ecuación de advección-difusión para $\rho$ en el de largo plazo y el límite a gran escala.

Ecuación macroscópica de la ecuación cinética con término fuente.

El análisis de multi-escala, conocido como el análisis de Chapman-Enskog, se utiliza para verificar si la ecuación cinética es consistente con la ecuación de advección a nivel macroscópico.

Con el fin de obtener la formulación macroscópica de la EDP del LBM, es necesario separar formalmente diferentes escalas de tiempo. Para este propósito, las variables no conservados, es decir, u y $\mathrm{P}$, se expanden alrededor de sus valores de equilibrio en términos de $\tau$. La modelación determinística se basa en la formulación y solución de ecuaciones diferenciales. En los modelos determinísticos de procesos de precipitación se usan ecuaciones diferenciales obtenidas de balances de materia en donde se asume que los tamaños de los cristales son cantidades continuas. Pero a escala microscópica los modelos determinísticos no son necesariamente válidos ya que los valores de las propiedades observables vienen del promedio tomado sobre los componentes microscópicos del sistema, de hecho la modelación determinística de un sistema con un número finito de elementos implicaría dos simplificaciones poco realistas: que los fenómenos que allí ocurren son continuos, y que los sistemas que parten de las mismas condiciones con iguales perturbaciones se comportan de la misma manera. Los materiales en esta parte de la investigación corresponden a los sistemas computacionales, para lo cual se viene utilizando un programa computacional a través del Matlab.

El método corresponde al desarrollo del balance de materia para el proceso de precipitación considerando las etapas de nucleación (nuc), agregación (agre) y rotura(rot), en donde se establece un sistema de ecuaciones diferenciales en derivadas parciales para los cuales se plantea su solución utilizando Método Reticular de Boltzmann.

La ecuación reticular de Boltzmann (LBEM) a diferencia de los métodos numéricos tradicionales basados en la discretización de las ecuaciones macroscópicas del continuo (conservación de la masa y Navier-Stokes), los LBEM se fundamentan en modelos microscópicos y mesoscópicos de las ecuaciones cinéticas.

Para poder establecer la solución numérica mediante un lenguaje de programación, es necesario establecer las ecuaciones del balance poblacional, que se derivan fundamentalmente, del fenómeno de precipitación.

Dentro de las ecuaciones macroscópicas resta plantear, las ecuaciones en términos del cambio de momento como:
Momento 0:

$$
\frac{\partial \rho}{\partial t}+\frac{\partial(\rho u)}{\partial r}=F
$$

Primer momento:

$$
\frac{\partial(\rho u)}{\partial t}+\frac{\partial P}{\partial r}=-\frac{1}{\tau}(\rho u-\rho v)
$$

Segundo momento:

$$
\frac{\partial P}{\partial T}=-\frac{1}{\tau}\left(P-P^{e q}\right)
$$

Con el fin de obtener la formulación macroscópica de la ecuación diferencial en derivadas parciales del Método Reticular de Boltzmann, es necesario separar formalmente diferentes escalas de tiempo. Para este propósito, las variables no conservados, es decir, u y $\mathrm{P}$, se expanden alrededor de sus valores de equilibrio en términos de $\tau$. El operador derivado en el tiempo también se expande en términos de $\tau$ como sigue:

$$
\begin{gathered}
u=v+\tau u^{(1)}+\tau u^{(2)} \\
P=P^{e q}+P^{(1)}+\tau P^{(2)} \\
\frac{\partial}{\partial t}=\frac{\partial^{(0)}}{\partial t}+\tau \frac{\partial^{(1)}}{\partial t}
\end{gathered}
$$

donde la velocidad de advección $\vee$ es el valor de equilibrio de la velocidad media local de u y los superíndices dentro del paréntesis denotan el orden de la expansión.

Planteadas estas preciciones, queda desarrollar la estrategia metodológica para realizar la programación. El principio que guía los LBEM es la construcción de un sistema dinámico en una retícula simple y simétrica (en su mayoría cuadrados en 2D y en 3D cúbicos) el cual involucra una serie de cantidades que pueden ser interpretadas como la pdf de partículas ficticias sobre los vínculos de la retícula. Estas cantidades luego evolucionan en un tiempo discreto según ciertas reglas que se seleccionan para lograr un comportamiento macroscópico deseable que surge con relación a las grandes escalas con el espaciado reticular.

El argumento básico para el uso de estos tipos de métodos cinéticos simplificados en la simulación de flujos de fluidos macroscópicos es el de que la dinámica macroscópica de un fluido es el resultado de la conducta colectiva de muchas partículas microscópicas en el sistema y que la dinámica macroscópica no es sensible a los detalles subyacentes en la física microscópica. Mediante el desarrollo de una versión simplificada de las ecuacio- 
nes cinéticas complejas, como lo es la ecuación de Boltzmann, es posible evitar que sigue a cada partícula como en simulaciones de dinámica molecular.

\section{Resultados}

\section{Parámetros experimentales}

Para el modelamiento se han considerado los resultados de un proceso por lotes con nucleación y crecimiento, para la obtención del nitrato de potasio publicados por Gunawan R. y otros (2004), en "High resolution algorithms for multidimensional population balance equations" donde la tasa de crecimiento es una función de las variables de tiempo variable tamaño y; en este caso, la concentración de la solución y la temperatura. Los parámetros cinéticos considerados fueron:

TABLA 2 Parámetros cinéticos

\begin{tabular}{cll} 
Variable & Valor & Unidades \\
\hline$b$ & 1.78 & Adimensional \\
$k_{b}$ & $4.64 \times 10^{-7}$ & Partícula $/ \mu \mathrm{m}^{3} / \mathrm{s}$ \\
$g$ & 1.32 & adimensional \\
$k_{g}$ & $1.16 \times 10^{2}$ & $\mu \mathrm{m} / \mathrm{s}$
\end{tabular}

Referencia: Gunawan y otros (2004).

La densidad del cristal, $\rho_{-} c$

$$
\rho_{c}=2.11 \times 10^{-12} \mathrm{~g} / \mu \mathrm{m}^{3}
$$

Y, la concentración de la solución saturada es:

$$
c=1.721 \times 10^{-4} T^{2}-5.88 \times 10^{-3} T+0.1286
$$

Los momentos de iniciación fueron evaluados analíticamente para una distribución inicial:

$$
f(L, 0)=\left\{\begin{array}{c}
-3.48 \times 10^{-4} L^{2}+0.136 L-13.3 \\
180.5 \mu m \leq L \leq 210.5 \mu m \\
0
\end{array}\right.
$$

La solución numérica lo desarrolla utilizando el método de momentos.

Estos mismos parámetros fueron utilizados en la publicación de Qamar S. y Mubeen S. (20|4), aplicando el método de características, para la solución numérica de la ecuación de balance poblacional.

El carbonato de calcio producido por la precipitación se utiliza en aplicaciones de pasta de papel y en los productos farmacéuticos por una variedad de técnicas de producción y aplicaciones. La importancia comercial generalizada también ha hecho que sea uno de los sistemas de precipitación más estudiados. Para la simulación se considera un reactor de precipitación continua con un posible objetivo de ser optimización en línea dinámica. Esto requiere soluciones rápidas y estables, pero con las limitaciones adicionales de la tecnología de medición y los parámetros del modelo, no se requiere una gran precisión. El examen de la viabilidad de simulación dinámica se hará examinar el equilibrio entre precisión y velocidad.

Tabla 3 Parámetros cinéticos para el $\mathrm{CaCO} 3$

\begin{tabular}{ccl} 
& Valor & \multicolumn{1}{c}{ Fuente } \\
$B_{0}$ & $1.5 \pm 0.2 \times 10^{8} \# / \mathrm{m}^{3} * s$ & Lynn, C. , 1989, \\
$\mathrm{G}$ & $6.7 \pm 0.1 \times 10^{-9} \mathrm{~m}^{2} / \mathrm{s}$ & Lynn, C. , 1989, \\
$\mathrm{G}$ & $1.28 * 10^{-10} * r h o^{2}$ & Collier, A. P., 1999. \\
$B_{0}$ & $5.29 \times 10^{-4} \mathrm{~m}^{2}$ & Schnebelen M., \\
$\mathrm{G}$ & $0.52 \pm 0.03 \times 10^{-9} \mathrm{~m}^{2} / \mathrm{s}$ & Andreassen, J.P., 2001 \\
$B_{0}$ & $1.68 * 10^{-10} \mathrm{~m}^{3} / \mathrm{h}$ & Zauner, R. , 1994, \\
$\mathrm{G}$ & $8.06 * 10^{-9} \mathrm{~kg}$ & Wszelaka, ,2014, \\
$B_{0}$ & $1 * 10^{-7} \mathrm{kn}$ & Wszelaka, 2014, \\
$\mathrm{C}$ & 0 & Vidal D. y otros \\
$\mathrm{T}$ & 1 & Vidal D. y otros
\end{tabular}

Complementa la ecuación del balance poblacional para la fase de distribución las ecuaciones diferenciales ordinarias (EDOs) para las variables continuas de fase. En el sistema de carbonato de calcio una serie de reacciones se producen entre especies en la fase líquida (Gandhi y otros., 1995).

Considerando la formación del carbonato de calcio a partir de hidróxido de calcio con dióxido de carbono disuelto. La reacción general está dada por:

$$
\mathrm{Ca}(\mathrm{OH})_{2}+\mathrm{CO}_{2} \rightarrow \mathrm{CaCO}_{3}+\mathrm{H}_{2} \mathrm{O}
$$

La precipitación ocurre cuando iones de calcio e iones de carbonato están presentes en proporciones tal que el producto de solubilidad del carbonato de calcio está en exceso. Las siguientes reacciones son importantes en el sistema:

$$
\begin{gathered}
\mathrm{Ca}(\mathrm{OH})_{2}(\mathrm{ac}) \rightarrow \mathrm{Ca}^{2+}+2 \mathrm{OH}^{-} \\
\mathrm{CO}_{2}+\mathrm{OH}^{-} \rightarrow \mathrm{HCO}_{3}^{-} \\
\mathrm{CO}_{2}+\mathrm{H}_{2} \mathrm{O} \rightarrow \mathrm{HCO}_{3}{ }^{-}+\mathrm{H}^{+} \\
\mathrm{Ca}^{2+}+\mathrm{CO}_{3}{ }^{2-} \rightarrow \mathrm{CaCO}_{3}(\mathrm{ac}) \\
\mathrm{HCO}_{3}{ }^{-} \rightarrow \mathrm{H}^{+}+\mathrm{CO}_{3}{ }^{2-} \\
\mathrm{H}_{2} \mathrm{CO}_{3} \rightarrow \mathrm{H}^{+}+\mathrm{HCO}_{3}^{-} \\
\mathrm{Ca}^{2+}+\mathrm{HCO}_{3}^{-} \rightarrow \mathrm{CaHCO}_{3}^{+} \\
\mathrm{Ca}^{2+}+\mathrm{OH}^{-} \rightarrow \mathrm{CaOH}^{+} \\
\mathrm{H}_{2} \mathrm{O} \rightarrow \mathrm{H}^{+}+\mathrm{OH}^{-}
\end{gathered}
$$


Las reacciones anteriores, contiene 12 especies:

Mediante la eliminación de Gauss el rango de la matriz es 7.

El análisis anterior es válido para el cálculo de la sobresaturación antes del nacimiento del primer núcleo. Después de que el crecimiento de la fase de partículas a expensas de los solutos en la fase líquida tiene que tenerse en cuenta en el balance de masa de las especies. Por supuesto, el método de análisis de las reacciones será inalterada.

Para simplificar, se consideran la alimentación para ser hidróxido de calcio y ácido carbónico. Este último no es físicamente razonable, pero puede considerarse equivalente a una determinada tasa de disolución de dióxido de carbono. La suposición de equilibrio entre las reacciones en fase líquida permite que el estado puede definirse con dos variables, concentraciones $\mathrm{Cco} 2 / 3$ carbonato total y $\mathrm{Cca} 2+$, elegidos porque son invariantes respecto a las reacciones en fase líquida. Sus balances son:

$$
\begin{aligned}
\frac{d}{d t} y-\sum_{i} F_{i} y_{i} & +D y= \\
& \propto \int_{0}^{\infty} d l s_{v} l^{2} G(l, y) n(l, t) \\
& =G_{t}(\boldsymbol{y}) \boldsymbol{G}^{T} \boldsymbol{n}(t)
\end{aligned}
$$

Donde $\mathrm{G}$ se define como el operador de crecimiento.

Las concentraciones de reactivos y de ésta sobresaturación se calculan utilizando relaciones de equilibrio para todas las reacciones en fase continua. La dinámica de crecimiento es del orden de horas en lugar de segundos, lo que permite la suposición de equilibrio. Los coeficientes de actividad se establecen mediante el modelo de Pitzer, y que la programación y comparación con los datos experimentales se desarrollan en el anexo, y cuyos resultados se presentan en la Figura.

\section{COEFICIENTE DE ACTIVIDAD DEL CLORURO DE CALCIO}

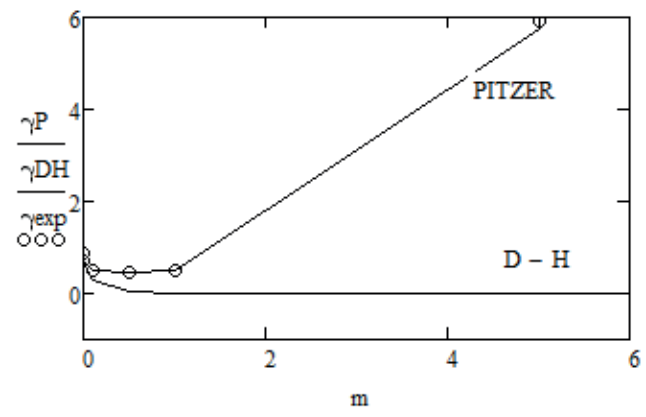

Figura I Determinación del coeficiente de actividad mediante de método de Pitzer

El modelo de Pitzer como se aprecia en la figura, representa muy bien los datos experimentales a bajas y altas concentraciones. El modelo de Debye-Hückel representado en la figura por la línea marcada con D-H puede ser utilizado sólo a concentraciones muy bajas, normalmente para fuerzas iónicas hasta $0,01 \mathrm{~mol} / \mathrm{kg}$.

La precipitación como un proceso de cristalización reactiva es compleja tanto experimentalmente como analíticamente. En las referencias sobre investigaciones experimentales para la precipitación aplicando la ecuación del balance poblacional se cuenta con los estudios realizados para el carbonato de calcio por Mahoned A. y Ramkrishna D. en "Efficient solution of population balance equations with discontinuities by finite elements", el cual incluye en su estudio la determinación del coeficiente de actividad mediante la corrección de Davies, en el presente trabajo de investigación, como lo hemos manifestado, se propone el modelo de Pitzer, el cual permite determinaciones del coeficiente de actividad a concentraciones mayores de $0.01 \mathrm{M}$. Tai C. y Chen P. (19995) en "Nucleation, Agglomeration and cristal morphology of calcium carbonate" realizan la precipitación mediante el método (MSMPR) (Mixed suspension mixed product removal crystallization). la precipitación del sulfato de bario se cuenta con datos experimentales de Kamyabi, M. y otros (20I2) en "CFD modeling of barium sulfate nano particles crystallization in a tank reactor by using population balance equations". Condiciones usadas para la simulación del crecimiento y nucleación.

$$
\begin{gathered}
a=\frac{1}{2} \quad b=5 \quad c=\frac{10}{3} \quad d=2 \\
G(l)=1-\operatorname{aexp}\left[-\frac{1}{d}\right] \\
b[t]=1-a
\end{gathered}
$$

Modelos nominales y parámetros para la simulación de la precipitación del carbonato de calcio.

$$
\begin{aligned}
& G(l, \sigma)=1.28 \times 10^{-10} \sigma^{2} \mathrm{~m} / \mathrm{s} \\
& a\left(l, l^{\prime}, \sigma\right)=8.71 \times 10^{-6} G(l, \sigma) \frac{\mathrm{m}^{4}}{\mathrm{~s}} \\
& \beta(n, \sigma) \\
& =5.07 \\
& \times 10^{7} \sigma^{1.62} \rho \frac{\pi}{6} \int_{0}^{\infty} d l l^{3} n(l, t) \text { partículas } / \mathrm{s} \\
& \qquad D=\frac{1}{5 h} \\
& K_{s p}=4.7 \times 10^{-3} \mathrm{~mol}^{2} / \mathrm{m}^{6}
\end{aligned}
$$

Parámetros determinados experimentalmente, por Mohamey y Ramkrishna (2002) en "Efficient solution of population balance equations discontinuities by finite elements". 
Aplicación al sistema de precipitación del sulfato de potasio

La distribución inicial es:

$$
n(x, 0)= \begin{cases}100 & \text { para } 0.4 \leq x \leq 0.6 \\ 0.01 & \text { en otra parte }\end{cases}
$$

La expresión para la nucleación rígida como una función del tiempo es dada como:

$$
n(t, 0)=100+10^{6} \exp \left(-10^{4}(t-0.215)^{2}\right)
$$

Las partículas son asumidas para tener un crecimiento con la velocidad de crecimiento de $\mathrm{G}=1.0 \mu \mathrm{ms}^{-1}$ y e computo del dominio es tomado entre $0 \leq \mathrm{x} \leq 2.0 \mu \mathrm{m}$. Los parámetros para la conglomeración y rotura son tomados como:

$$
\begin{aligned}
a\left(x, x^{\prime}\right) & \left.=1.5 \times 10^{-5}\right) \\
b\left(x, x^{\prime}\right) & =2 / x^{\prime} \\
\Gamma(x) & =x^{2}
\end{aligned}
$$

Tiempo final, $\mathrm{tf}=0.5 \mathrm{~s}$

Puntos en la cuadrícula (grid points), N=200

Tolerancia absoluta, $10^{-8}$

Toleranacia relativa, $10^{-4}$

Kumar y Ramkrhisna (1996) en "On the solution of population balance equations by discretization - 1 . A fixed pivot technique", para la conservación de los números y la masa $\eta$ está dada por la expresión simple:

$$
\eta= \begin{cases}\frac{x_{i+1}-v}{x_{i+1}-x_{i}} & x_{i} \leq v \leq x_{i+1} \\ \frac{v-x_{i-1}}{x_{i}-x_{i-1}} & x_{i-1} \leq v \leq x_{i}\end{cases}
$$

$\mathrm{v}=$ Volumen de la partícula.

$\mathrm{xi}=$ Volumen representativo para el rango de tamaño $\mathrm{i}$ Majumber y otros (2012) establecen:

$$
\rho=\sum_{i=0}^{2} \widehat{f}_{l}+\frac{\Delta t}{2} F
$$

$\rho=$ Concentración de las especies transportadas, $g$ $\mathrm{G}^{\mathrm{m}} \mathrm{m}^{-3}$

$\mathrm{t}=$ Tiempo, $\mathrm{s}$

$\mathrm{fi}=$ Función distribución discreta de Boltzmann, \# $\mu_{\mathrm{m}}^{-1}$

$\widehat{\mathrm{f}}=$ Función de distribución discreta de Boltzmann re-definida, $\# \mu \mathrm{m}^{-1}$

La ecuación de advección

$$
\frac{\partial \rho}{\partial t}+\frac{\partial(v \rho)}{\partial r}=F
$$

$\rho=$ Concentración de las especies transportadas,

$\mathrm{g} \mu \mathrm{m}-3$

$\mathrm{v}=$ Velocidad de advección

$\mathrm{F}=$ Termino fuente.

\section{Distribución del Tamaño de Cristales}

Tomando como referencia el código en Matlab desarrollado por Majumder A. y Kariwala de la School of Chemical and Biomedical Enggineering, Nanyang Technological University, Singapore. Date: September, 20 I I, utilizando el método Reticular de Boltzmann, cuyo resultado se representa en la siguiente gráfica:

\section{DISTRIBUCION POBLACIONAL DEL CARBONATO DE CALCIO}

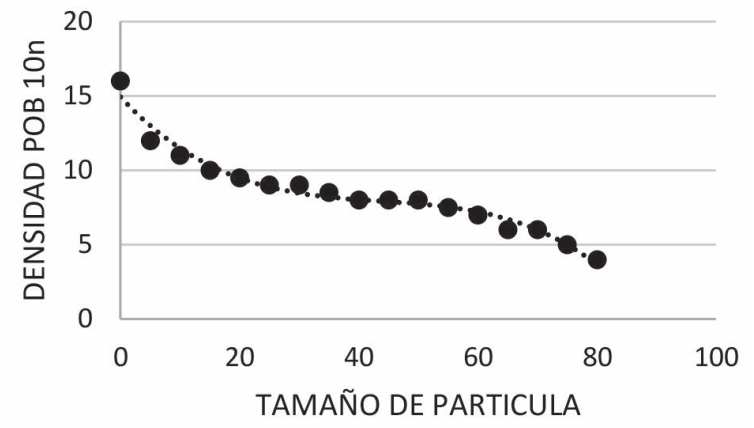

\section{Discusión}

El modelamiento en Ingeniería Química, se refiere a la sistematización el cual depende de los valores metodológicos y cognitivos como una herramienta fundamental para entender, analizar, diseñar, operar, controlar los procesos reales Hulburt y Katz, en Ingeniería Química, formularon la ecuación de Balance poblacional usando las ideas de mecánica estadística, mientras Randolph y Larson basado en su formulación sobre mecánica del continuo, describe los procesos de cristalización y precipitación los cuales se conocen también como cristalización reactiva.

Muchas técnicas numéricas como por ejemplo, residuos ponderados, método de los momentos, colocación ortogonal, colocación en elementos finitos han sido propuestos en la literatura durante muchos años para resolver las ecuaciones del balance poblacional en los procesos de precipitación, el método reticular de Boltzmann, es una nueva propuesta planteada por Majumber A., Kariwala V., Ansumali S., Rajendran A. (20/2) en "Latice Boltzmann method for population balance equations with simultaneous growth, nucleation, aggregation and breakage", mediante el cual se determina la distribución del tamaño de cristales con respecto a la longitud del cristal, el cual constituye uno de los parámetros más importantes en los procesos de cristalización reactiva, porque esto determina las características más importantes del producto para su comercialización, control de las operaciones, optimización del proceso y sobre todo 
para poder plantear nuevas posibilidades de procesos de precipitación tanto en los procesos productivos, como en la descontaminación de los efluentes por medio de una precipitación selectiva.

Como se muestra en la figura,

para una velocidad de partícula, c $=10$

Tiempo final, $\mathrm{Tf}=0.5$

Parámetro de relajación, $\beta=0.96$

Número de puntos de cuadrícula, $\mathrm{N}=200$,

Y considerando los parámetros presentados en los resultados, la gráfica desarrollada con el método reticular de Boltzmann se aproxima a los valores analíticos, considerando el tamaño en $\mu m$ y la distribución del tamaño de cristales (CSD) en $\# / \mu \mathrm{m}$.

\section{Conclusiones}

La ecuación del balance poblacional en una dimensión ( I D) es desarrollada para un proceso de precipitación o cristalización reactiva, considerando el crecimiento, la nucleación, la agregación y la rotura.

El Método Reticular de Boltzmann resuelve el sistema de ecuaciones del balance poblacional el cua ha sido contrastado con los modelos analíticos para la precipitación del nitrato de potasio.

\section{Referencias}

Galbraith S. (20|4). Modelling and simulation of inorganic precipitation with nucleation, cristal growth and aggregation: anew approach to an old method.

Majumder y otros (20/2). Lattice Boltzmann method for population balance equations with simultaneous growth, nucleation, aggregation and breakage. Chemical Engineering Science, 69, págs. 316-328.

Qamar S, Mubeen S. (20I4). Application of the method of characteristics to Population Balance Models considering growth and nucleation phenomena. Applied Mathematics, vol. 5, pág. |853-|862. 